\title{
Caracterización clínica y neurofisiológica del síndrome de Guillain-Barrè en el Hospital Escuela
}

\author{
Carmen J. Aguilar*, Marco T. Medina*, Rebeca Hernández*, Claudia Amado*r, \\ Martha Matamoros*, José A. Lara Pinto*
}

\section{RESUMEN}

\section{Antecedentes y Objetivos}

En Honduras se realizó entre 1989-1999 un estudio que valoró la incidencia de Guillain-Barrè y parálisis flácida en una población pediátrica, en el hospital Materno Infantil obteniendo valores de 1.37/100,000 casos por año para Síndrome de Guillain-Barrè; por otro lado de 1.89/100,000 habitantes por año de parálisis flácida a nivel nacional y hospitalaria. La incidencia fue considerada alta en comparación con otros estudios. El objetivo del presente estudio fue investigar los subtipos clínicos y neurofisiológicos de pacientes con SGB en una población hondureña pediátrica y adulta en Honduras comparado con un grupo control sano.

\section{Materiales y Métodos}

Este es un estudio transversal, analítico con pacientes consecutivos siguiendo los criterios de Asbury et al., en pacientes de 2 a 55 años de edad en el Hospital Escuela y Materno Infantil en el período de 1 febrero 2007 a Agosto 2008, se estudiaron 29 pacientes y 58 controles sanos. Se les realizó la evaluación clínica y neurofisiológica. Se estudiaron los nervios motores Mediano, Ulnar, Peroneo, Tibial y las respuestas tardías onda $\mathrm{F}$ y reflejo $\mathrm{H}$; los nervios sensitivos Mediano, Ulnar y Sural con la técnica descrita por Parano E, Uncini A. etal (9).

* Carmen J. Aguilar,

Facultad de Ciencias Medicas, Postgrado de Neurología, Hospital Escuela

* Marco T. Medina,

Facultad de Ciencias Medicas, Postgrado de Neurología, Hospital Escuela

* Rebeca Hernández,

* ClaudiaAmador,

* Martha Matamoros,

Servicio de Cuidados Intensivos Pediátricos y Emergencia, Hospital Escuela

* JoséA. Lara Pinto,

Epidemiólogo 


\section{Resultados}

Las formas desmielinizantes se presentaron en 19 (65\%) pacientes y las formas axonales en 10 (35\%) de los casos. Estas últimas tuvieron la mayor severidad y discapacidad, así mismo 9 (90\%) de los casos requirieron asistencia en una Unidad de Cuidados Intensivos, y un significativo mayor número de días de hospitalización en relación a las formas desmielinizantes $\mathrm{p}=0.003$.

\section{Conclusiones}

Se encontró una alta incidencia de formas axonales del SGB, similar a los datos obtenidos en estudios realizados en el Norte de China y Japón. Presentando una mayor severidad, discapacidad y un incremento de los días de hospitalización en relación a las formas desmielinizantes.

\section{Palabras Clave: Guillain-Barrè, desmielinizante, axonal, electrofisiológico, electromiografía, parálisis}

\section{ABSTRACT}

Background and Objective. In Honduras, between 1989 and 1999, a study aiming to calculate the incidence of Guillain-Barré syndrome and flaccid paralysis in a pediatric population was developed in the Materno-Infantil Hospital. High compared to other studies, the ratio found at the time was 1.37/100.000 cases of Guillain-Barré syndrome per year and 1.89/100.000 cases of flaccid paralysis per year.

The objective of this study was to determine the clinical and neurophysiological subtypes found in patients with Guillain-Barré syndrome in a Honduran pediatric and adult population, compared to a healthy control group.

\section{Materials and Methods}

A transversal analytical study with consecutive patients following the criteria from Asbury at el was developed. The 29 patients, who were between the ages of 2 and 55 years, attended the Hospital Escuela and Materno Infantil during February 1, 2007, through August 2008. A clinical and neurophysiological evaluation was performed to the patients and the 58 healthy individuals. Motor function was assessed to the median, ulnar, peroneal, and tibial nerves, as well as the late F-wave response and the $\mathrm{H}$ reflex. The sensitive function of the median, ulnar, and sural nerves was evaluated following the technique described by Parano E, UnciniA. et al (9). 


\section{Results}

The demyelinating forms presented in 19 patients (65\%); the axonal forms in 10 (35\%). The former had greater severity and generated more incapacity, 9 of them requiring attention in the Intensive Care Unit resulting in more bed days, compared to the demyelinating forms $(p=0,003)$.

\section{Conclusions}

Similar to studies held at Northern China and Japan, a high incidence of the axonal forms of Guillain-Barré syndrome was found, presenting a greater severity and more bed days compared to the demyelinating forms.

Key Words: Guillain-Barré Syndrome, demyelinating, axonal, electrophysiological, electromyography, palsy. 


\section{INTRODUCCIÓN}

El síndrome de Guillain-Barrè (SGB) fue descrito en 1916 por Guillain, Barrè y Strohl en dos soldados que presentaron una parálisis arrefléctica aguda (1), seguida de recuperación. Basados en estudios neurofisiológicos extensos sobre SGB se han identificado diferentes subtipos, que presentan un patrón específico con diferentes características clínicas, fisiológicas, serológicas, patológicas y presumiblemente etiológicas: polineuropatía desmielinizante inflamatoria aguda (AIDP), neuropatía axonal motora aguda (AMAN)), neuropatía axonal sensitiva motora aguda(AMSAN), Síndrome de Fisher y otras variantes menos características (2). En el Norte de China y Japón se ha encontrado una alta incidencia de formas axonales hasta en un 65\% en algunas series, asociadas a procesos infecciosos gastroentéricos principalmente por Campylobacter jejuni. Este estudio se diferencia con otros estudios realizados en el país en la realización de un grupo de 2 casos controles por cada caso a quienes se realizó protocolo de polineuropatía, teniendo un total de 29 casos y 58 controles.

En Honduras se realizó entre 1989-1999 un estudio que valoró la incidencia de Guillain-Barrè y parálisis flácida en una población pediátrica, en el hospital Materno Infantil obteniendo valores de 1.37/100,000 casos por año para Síndrome de Guillain-Barrè y de 1.89 en 100,000 habitantes por año de parálisis flácida a nivel nacional y hospitalaria (3). La incidencia fue considerada alta en comparación con otros estudios.

Clínicamente el SGB se ha caracterizado por parálisis simétrica ascendente, y el cumplimiento de los criterios de Asbury et al revisados de 1990 (4). Se han alcanzado mayores avances en relación al entendimiento de los mecanismos de algunos de los subtipos.

Existe fuerte evidencia de que los subtipos axonales de SGB, neuropatía axonal motora aguda (AMAN), y neuropatía motora y sensitiva axonal son causadas por anticuerpos a gangliósidos en el axolema que se dirigen a los macrófagos a invadir el axón hasta el nodo de Ranvier (5) y se han relacionado con proceso infecciosos gastroentéricos principalmente por Campylobacter jejuni (6) por lo que es de mucho interés investigativo realizar estudios neurofisiológicos que ayuden a diferenciar los subtipos axonales y desmielinizantes para de esta manera valorar pronóstico y terapias de rehabilitación a largo plazo en pacientes adultos así como en niños.

EI SGB puede ser discapacitante a largo plazo por lo tanto es importante determinar qué tipo de síndrome se está presentando con mayor frecuencia y qué pronóstico a corto y largo plazo se ofrece a los pacientes en los casos desmielinizantes y 
axonales en nuestra población(7).

El objetivo de este estudio es investigar los subtipos clínicos y neurofisiológicos de pacientes con SGB en una población pediátrica y adulta en Honduras comparado con un grupo control sano.

\section{METODOLOGÍA}

\section{Diseño metodológico y período de estudio}

Se diseñó un estudio transversal, analítico con pacientes consecutivos siguiendo los criterios de Asbury et al en pacientes dentro del rango de 2 a 55 años de edad. Se captaron los pacientes menores de 18 años del Bloque Materno Infantil del Hospital Escuela y los mayores de 18 años del Bloque Médico Quirúrgico (Departamento de Medicina Interna) que ingresaron con sospecha de SGB, en el período comprendido entre el 1 de Febrero del 2007 al 30 de Agosto del 2008 (19 meses), de forma consecutiva, para un total 29 enfermos y 58 controles.

Los pacientes fueron tomados de los servicios de salas de Pediatría, Medicina Interna y Unidades de Cuidados Intensivos, a quienes se realizó la evaluación clínica, epidemiológica y neurofisiológica entre los 7 a 30 días de evolución. Esta última con un equipo Cadwell Sierra II Wedge 0104sw001010. Se realizó estudio de los nervios motores Mediano, Ulnar, Peroneo, Tibial y las respuestas tardías onda F y reflejo $\mathrm{H}$; los nervios sensitivos Mediano, Ulnar y Sural con la técnica descrita por Parano E, Uncini $A$. et al publicados en el libro de referencia Electromyography and Neuromuscular Disorders 2005, David C. Preston y Barbara E. Shapiro (8).

Para el análisis estadístico se utilizó el paquete Epi Info, versión 3.5.1.

Se captaron los casos moderados y severos en vista de que fueron los que permanecieron más días hospitalizados.

Para la recolección de datos se realizó exploración clínica, epidemiológica y neurofisiológica. Se aplicó instrumento tipo encuesta, previo consentimiento informado, autorizando los familiares se realizara el estudio neurofisiológico a cada paciente. Se usaron los criterios neurofisiológicos presentados en la tabla 1. (9)

\section{Lugar de Estudio}

El Hospital Escuela: Bloque Materno Infantil y Médico quirúrgico es el centro 
hospitalario de referencia nacional más grande con que cuenta el sistema de salud pública. La población de su área de influencia es alrededor de un $40 \%$ del total de los hondureños (3,200,000 habitantes). Estos cuentan con dos Unidades de Cuidados Intensivos que son vitales para el manejo del paciente con parálisis flácida que compromete los músculos respiratorios, aunque aún los cupos de dichas unidades se tornan insuficientes para las exigencias de la población.

Tabla 1. Criterios para polineuropatías desmielinizantes y axonales

\begin{tabular}{|l|c|}
\hline $\begin{array}{c}\text { Criterios para Polineuropatía } \\
\text { desmielinizante inflamatoria aguda }\end{array}$ & $\begin{array}{c}\text { Criterios para formas axonales de } \\
\text { Síndrome de Guillain-Barrè: }\end{array}$ \\
\hline $\begin{array}{l}\text { Los pacientes deben mostrar al menos } \\
\text { una de las siguientes características en } \\
\text { dos o más nervios durante las primeras } \\
\text { dos semanas de enfermedad: }\end{array}$ & $\begin{array}{c}\text { a. No evidencia de desmielinización } \\
\text { (como fue definido arriba). }\end{array}$ \\
$\begin{array}{l}\text { a. Velocidad de Conducción <95\% del } \\
\text { límite inferior de normalidad (LIN) si } \\
\text { la amplitud }<80 \% \text { del límite inferior de } \\
\text { normalidad. }\end{array}$ \\
$\begin{array}{l}\text { velocidad de conducción <85\% del } \\
\text { LIN sila amplitud es }<50 \% \text { del LLN. }\end{array}$ \\
$\begin{array}{l}\text { b. Latencia distal }>110 \% \text { del límite } \\
\text { superior de normalidad (LSN) si la } \\
\text { amplitud es normal; latencia distal } \\
<120 \% \text { del LSN si la amplitud es } \\
\text { menor que el LIN }\end{array}$ \\
$\begin{array}{l}\text { c. Evidencia de dispersión temporal } \\
\text { inequívoca. }\end{array}$ \\
$\begin{array}{l}\text { d. Latencia de la respuesta F }>120 \% \\
\text { del LSN. }\end{array}$ \\
\hline
\end{tabular}

\section{Muestra poblacional}

Se estudiaron los pacientes consecutivos menores de 18 años que ingresaron con parálisis flácida al Bloque Materno Infantil y la población mayor de 18 años 
ingresados en el Bloque Médico Quirúrgico del Hospital Escuela en el período mencionado. Durante este período del 1 Febrero del 2007 al 30 de Agosto del 2008, se ingresaron un total de 61 pacientes de los cuales 32 fueron excluidos por no llenar los criterios de inclusión ya que se les clasificó como SGB con un grado de debilidad leve y captados después de los primeros 5 días de evolución que fueron dados de alta de forma temprana y remitidos al Hospital General San Felipe para su seguimiento.

Tabla 2. Criterios de Inclusión y exclusión para participar en el estudio

\begin{tabular}{|c|c|}
\hline Criterios de inclusión & Criterios de exclusión \\
\hline - Tener entre 7 a 30 días de & - Pacientes con uroporfobilinógeno \\
evolución de inicio de la debilidad. & positivo o débil positivo. \\
- Cumplir con los criterios adap- & - Pacientes con disminución en \\
tados de Asbury y Cornblath, 1990. & niveles de potasio que presentaron \\
- Aceptar participar en el estudio de & $\begin{array}{l}\text { recuperación de la debilidad al } \\
\text { reponer potasio. }\end{array}$ \\
forma voluntaria previo consenti- & - Antecedentes de debilidad mus- \\
miento informado. & cular crónica o enfermedades de \\
& tiponeuropatías crónicas. \\
& - Antecedentes de SGB. \\
& - Presencia denivel sensitivo \\
\hline
\end{tabular}

\section{Aspectos Bioéticos}

Se brindó información a cada paciente y sus familiares acerca del estudio a realizar, obteniendo su consentimiento informado para poder realizar la exploración clínica y neurofisiológica de cada paciente, haciéndoseles saber que no participar en el estudio no influía en la calidad de la atención médica brindada y asegurándoles la confidencialidad de la información obtenida.

\section{Recolección de datos intra-hospitalarios}

Se recolectaron datos generales, socioeconómicos, antecedentes médicos del paciente y familiares, tiempo de evolución, se obtuvieron datos epidemiológicos acerca del mes de presentación de la enfermedad, se realizó recolección de datos de cada estudio neurofisiológico realizado con equipo marca Cadwell Sierra, clasificando cada paciente en subtipos según los criterios electrodiagnósticos 
para Polineuropatía desmielinizante inflamatoria aguda en conducción de nervios motores y criterios para Formas axonales de SGB.

\section{Operacionalización de variables}

Se utilizaron los criterios de clasificación para polineuropatía desmielinizante aguda y formas axonales de SGB basados en datos de la Clinica Mayo publicados por Kelly; así como los de Albert et al $(7,8)$ sobre los criterios para el SGB (4). Para las variables clínicas se utilizaron los criterios adaptados de Asbury y Cornblath, 1990 (5). Para una mayor certeza de la clasificación se realizó una doble verificación diagnóstica por parte del grupo investigador y al momento de la tabulación de los datos neurofisiológicos obtenidos.

\section{Análisis Estadístico}

Inicialmente se realizó un análisis descriptivo de las variables cuantitativas y cualitativas comparando los resultados del grupo de trabajo con el grupo de comparación, se calcularon las medias, frecuencias, varianzas y p en función de determinar valores normales y tendencias de las variables, basados en un grupo control de dos por cada caso seleccionados por edad y sexo.

La matriz de datos fue construída en el paquete de Microsoft Office Excel 2003 y el análisis estadístico se realizó con el paquete Epi-Info versión 3.5.1 y se estableció una significancia estadística para valores de $p<0.05$.

\section{Resultados}

De una muestra de pacientes consecutivos durante el período de estudio de Febrero 2007 a Agosto del 2008 se ingresaron 61 casos de parálisis flácida con sospecha de Síndrome de Guillain-Barrè, en total entre ambos centros hospitalarios. De estos se eliminaron 32 casos por no llenar los criterios de inclusión o tratarse de casos leves que no requirieron más de 5 días de hospitalización. Todos los pacientes participaron en el estudio previo consentimiento informado.

\section{Descripción epidemiológica}

De los 29 casos ( $n=29$ ) estudiados la edad media fue 21 años, con un rango de edad de 2 a 55 años. Del total de pacientes 12 (41\%)) fueron del sexo femenino y 17 (58\%) fueron del sexo masculino. 
Del total de casos 13 fueron menores de 18 años y 16 mayores de 18 años. De los menores de 18 años 5 (38\%) presentaron formas axonales y 8 (62\%) desmielinizantes, observados en Tabla 3.

Tabla 3. Distribución de formas neurofisiológicas de SGB en edad pediátrica

\begin{tabular}{|c|c|c|c|}
\hline \multicolumn{4}{|c|}{ Distribución de formas axonales vrs desmielinizantes en edad pediátrica } \\
\hline Edad/Años & Axonal & Desmielinizante & \\
\hline 2 & 1 & 1 & 2 \\
\hline 3 & 1 & 0 & 1 \\
\hline 4 & 1 & 0 & 1 \\
\hline 6 & 0 & 1 & 1 \\
\hline 9 & 0 & 1 & 1 \\
\hline 11 & 0 & 1 & 1 \\
\hline 12 & 0 & 1 & 1 \\
\hline 15 & 1 & 2 & 3 \\
\hline 16 & 0 & 1 & 1 \\
\hline 17 & 1 & 0 & 1 \\
\hline Total & 5 & 8 & 13 \\
\hline$\%$ & $38 \%$ & $62 \%$ & $100 \%$ \\
\hline
\end{tabular}

De los pacientes menores de 18 años $(n=13), 7$ presentaron afectación de músculos respiratorios mientras que de los mayores de 18 años $(n=16), 12$ pacientes tuvieron afectación de músculos respiratorios.

La media de días de hospitalización entre los menores de 18 años fue de 30 días, mientras que en los mayores de 18 años fue 34 días.

La mayoría de los pacientes 13 (45\%) procedieron del Departamento de Francisco Morazán, 4 (14\%) de San Pedro Sula, Cortés y $3(10 \%)$ de los Departamentos de El Paraíso y Comayagua en igual proporción. En 24 (83\%) pacientes encontramos una o más de una necesidad básica insatisfecha. No se encontraron antecedentes de enfermedades inmunológicas ni de afectación neuromuscular en los casos ni en sus familiares.

En 23 (79\%) de los casos se encontró antecedente de un proceso infeccioso en los últimos 30 días, con presentación del antecedente de proceso infeccioso en 16 (55\%) casos de las formas desmielinizantes y en 7 (24\%) de las formas axonales. 
En $6(21 \%)$ de los casos no se encontró antecedente de un proceso infeccioso.

Los procesos infecciosos gastroentéricos se presentaron en 11 pacientes del total, de los que 7 eran menores de 18 años. Los procesos infecciosos respiratorios se observaron en 12 pacientes, de los cuales 7 eran mayores de 18 años.

Se encontraron asociados otros procesos infecciosos diferentes de gastroentérico y respiratorio en 2 casos uno de ellos fue varicela en un adulto de 43 años que cursó con un subtipo axonal y falleció; y un segundo caso con cuadro de Dengue grupo B con una variante desmielinizante. Los meses de mayor incidencia de casos de SGB fueron Febrero 4 (14\%), Mayo 4 (14\%), Julio 4(14\%) yAgosto 7 (17\%).

Se encontró un promedio general de hospitalización de 32 días. En los pacientes con formas desmielinizantes el promedio fue de 27 días y en las formas axonales de 36.7 días $p=0.03$

La forma desmielinizante (polirradiculoneuropatía desmie-linizante aguda) se presentó en 19 (65\%) de los pacientes y las formas axonales se presentaron en 10 (35\%) casos, con los subtipos neuropatía axonal motora aguda en un $8(28 \%)$ pacientes y la neuropatía axonal sensitiva motora aguda en 2 (7\%) casos.

El promedio de días de evolución al momento de realizar estudios neurofisiológicos fue de 17 días. En el 100\% de los pacientes se administró inmunoglobulinas de forma oportuna en los primeros 5 días de inicio de la enfermedad. Se presentó mayor número de pacientes con afectación de músculos respiratorios en los casos de Guillain-Barrè desmielinizantes.

Del total de los pacientes con subtipos axonales, 9 (90\%) requirieron estar en Unidad de Cuidados Intensivos, mientras que de los subtipos desmielinizantes 11 (57\%) casos necesitaron permanecer en Unidades de Cuidados Intensivos.

Las formas axonales tuvieron mayor afectación de músculos respiratorios presentándose en $9(90 \%)$ pacientes, mientras que en las formas desmielinizantes se presentó en $13(68 \%)$ de ellos.

De los casos menores de 15 años 8 (80\%) requirieron ser ingresados en Unidades de Cuidados Intensivos, mientras que en mayores de 15 años fueron 12 (63\%) pacientes. 
Figura 1. Estudio Neurofisiológico Nervio Mediano Motor

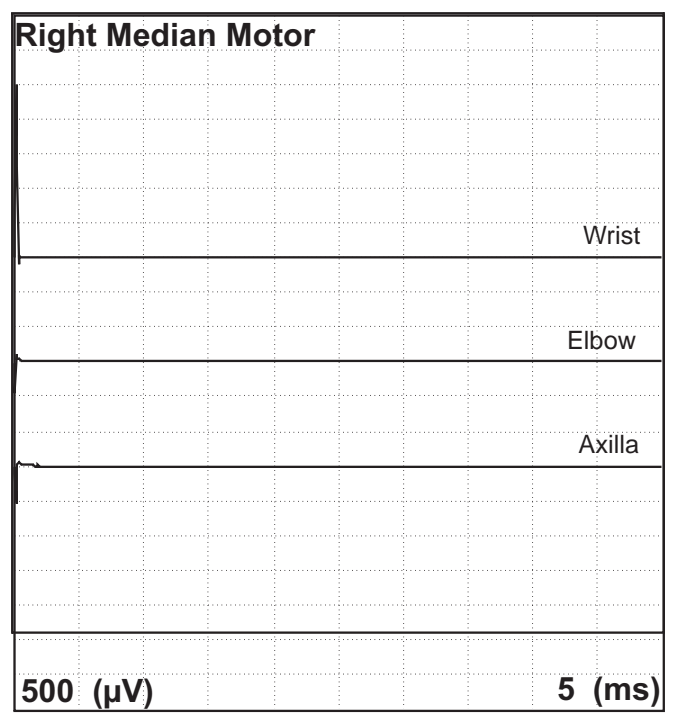

Nervio Mediana Motor en paciente son subtipo AMAN.

Se observa inexatibilidad en los tres sitios estimulados.

Figura 2. Respuesta tardía Onda F

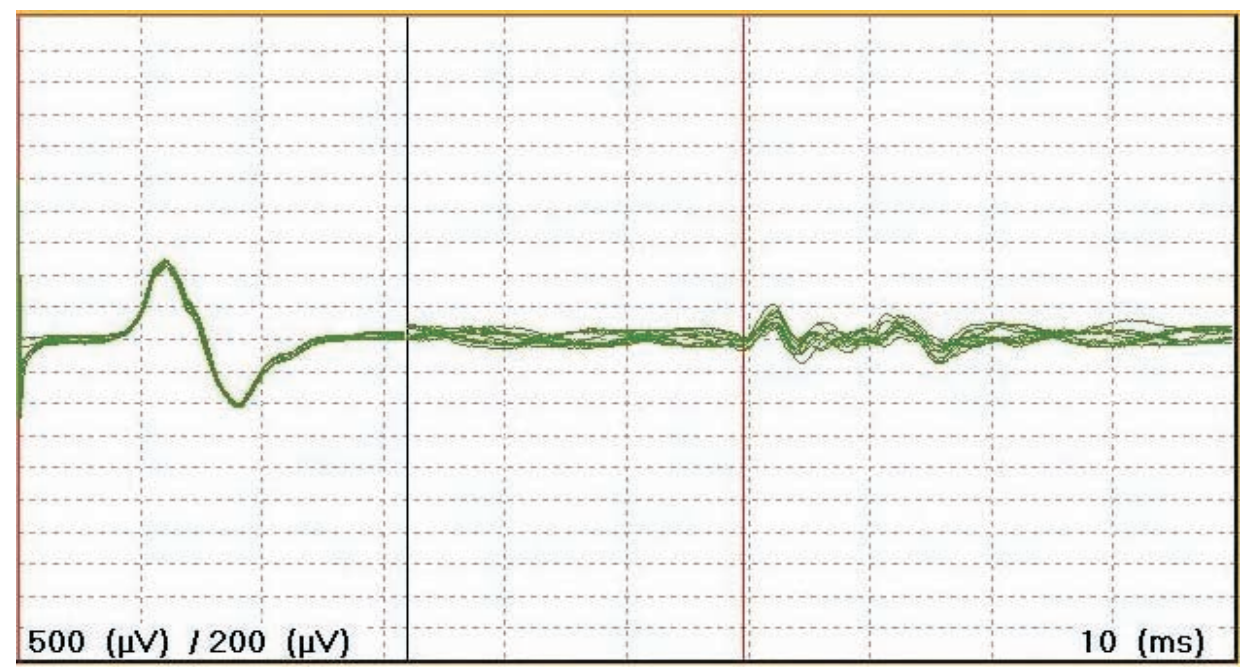

Estudio de la onda $\mathrm{F}$ de un paciente conSGB, donde se muestra latencia mínima de $\mathrm{F}$ prolongada. Forma desmielinizante. En las formas axonales fue inexcitable 
Figura 3. Estudio Neurofisiológico del nervio Mediano Sensitivo

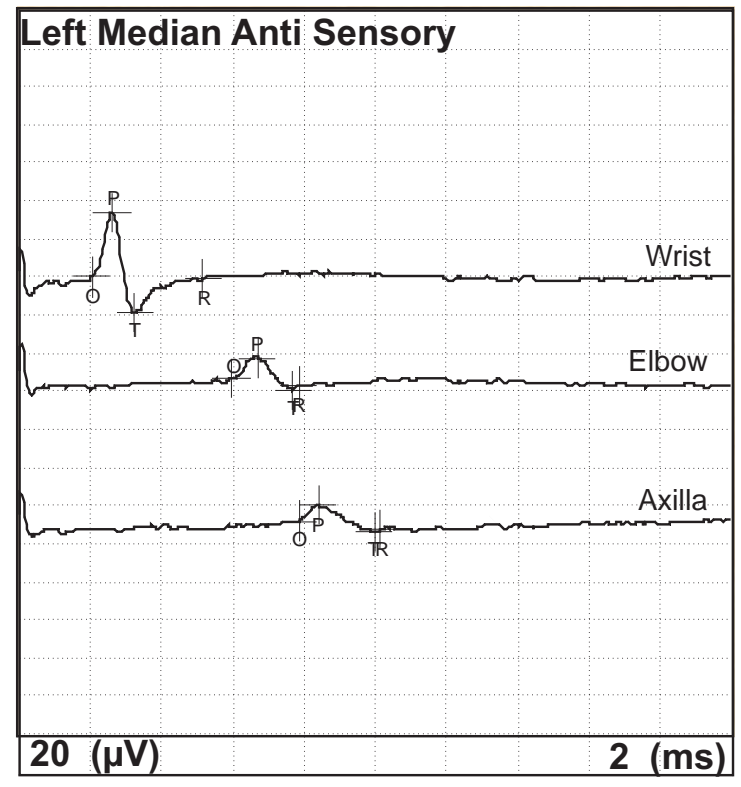

Ilustración del Nervio Mediano sensitivo en subtipo AMSAN; muestra disminución de las amplitudes a nivel codo y la axila

Se encontró tetraparesia en el $100 \%$ de los casos, presentándose inicio de la debilidad en miembros superiores en 9 (31\%) pacientes del total, que a su vez se presentó con mayor frecuencia en las formas desmielinizantes en número de 6 (67\%). El 100\% de los casos con inicio de la debilidad en miembros superiores fueron mayores de 15 años. En el resto de los pacientes estudiados predominó la debilidad en forma ascendente.

El grado de debilidad en los casos estudiados varió entre moderada 9 (31\%) a severa 20 (69\%).

La afectación de nervios craneales se presentó en forma indistinta en un 30\% de los casos desmielinizantes y axonales. En 9 casos (31\%) se encontró afectación del VII nervio craneal y en un caso (3\%) afectación del VI nervio craneal bilateral que se presentó en un niño de cuatro años de edad en quien se presentó además diplejía facial. Ningún caso mostró afectación esfinteriana.

La mayor severidad de la enfermedad se presentó en los casos axonales, de los que $9(90) \%$ fueron formas severas. El grado de discapacidad fue mayor en los casos 
axonales presentándose de forma severa en 8 pacientes (80\%).

Las complicaciones más frecuentes fueron las disautonomías presentándose en 21 (72\%) casos: las cuales fueron taquicardia: 21 (72\%); hipertensión arterial: 14 (48\%); seguida de Infecciones de Tracto Urinario: 13 (45\%); traqueítis:12 (41\%); complicaciones metabólicas: 9 (31\%) y atelectasia: 5 (17\%).

Del total de casos ( $n=29$ ) fallecieron 4 personas; de ellos uno de dos años femenino y tres mayores de 15 años dos masculinos y un femenino. El total de fallecidos requirió asistencia en Unidades de Cuidados intensivos, presentando complicaciones infecciosas pulmonares, infección de tracto urinario, alteraciones metabólicas importantes y disautonomías. Las figuras 1,2 y 3 se presentan como ejemplos de los estudios neurofisiológicos realizados a algunos de los pacientes. Muestran la afectación de las amplitudes y latencias. Desde el punto de vista neurofisiológico podemos comparar las latencias y velocidades en personas sanas versus los pacientes con las formas desmielinizantes y axonales lo que se observa en los gráficos 1 y 2 con los valores numéricos en las tablas 4 y 5 con sus respectivas $p$ de significancia

Gráfico 1. Gráfico de dispersión

Relación de parámetros de velocidad de conducción y latencia en nervio Mediano (motor)

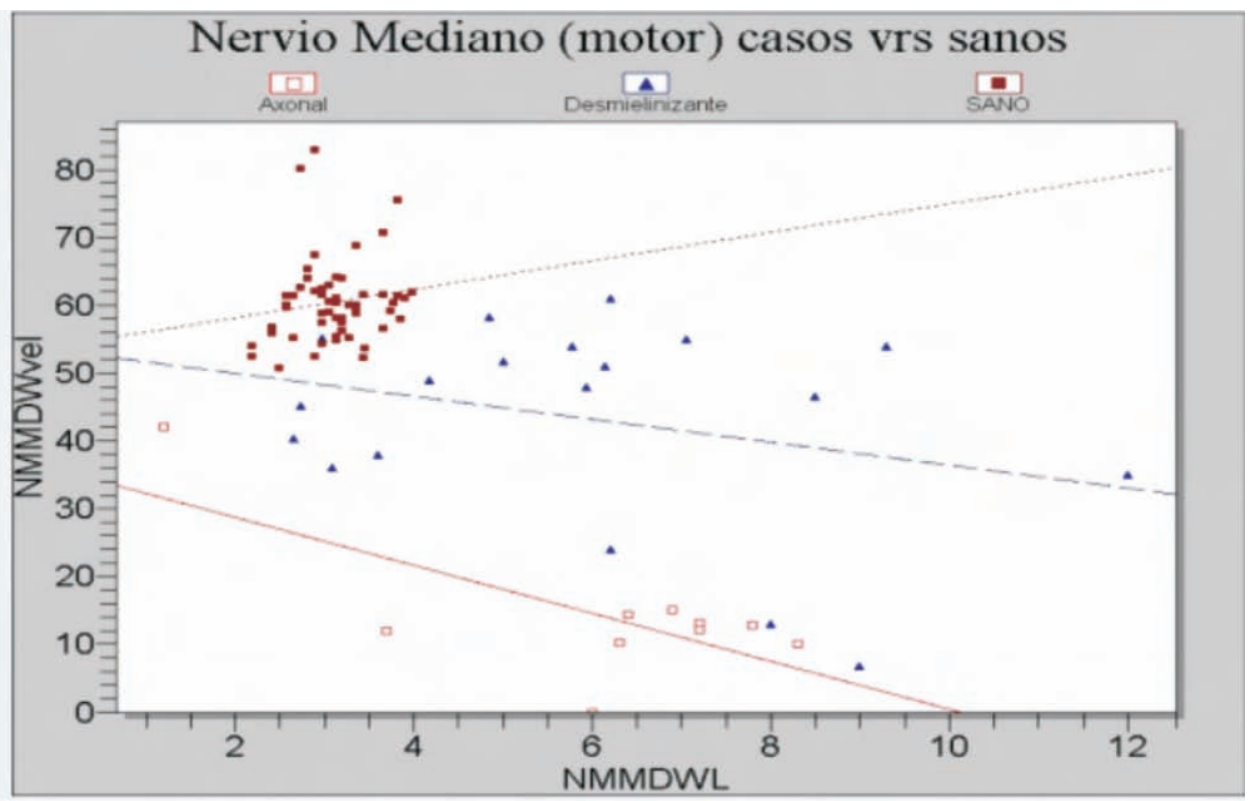


Se observan las formas desmielinizantes y axonales en comparación con los controles sanos. Las latencias y velocidad se encuentran afectadas con mayor claridad en las formas desmielinizantes y las amplitudes en las formas axonales en el nervio Mediano Motor

Grafico 2. Gráfico de dispersión

Relación de parámetros de velocidad de conducción y Latencia del Nervio Peroneo

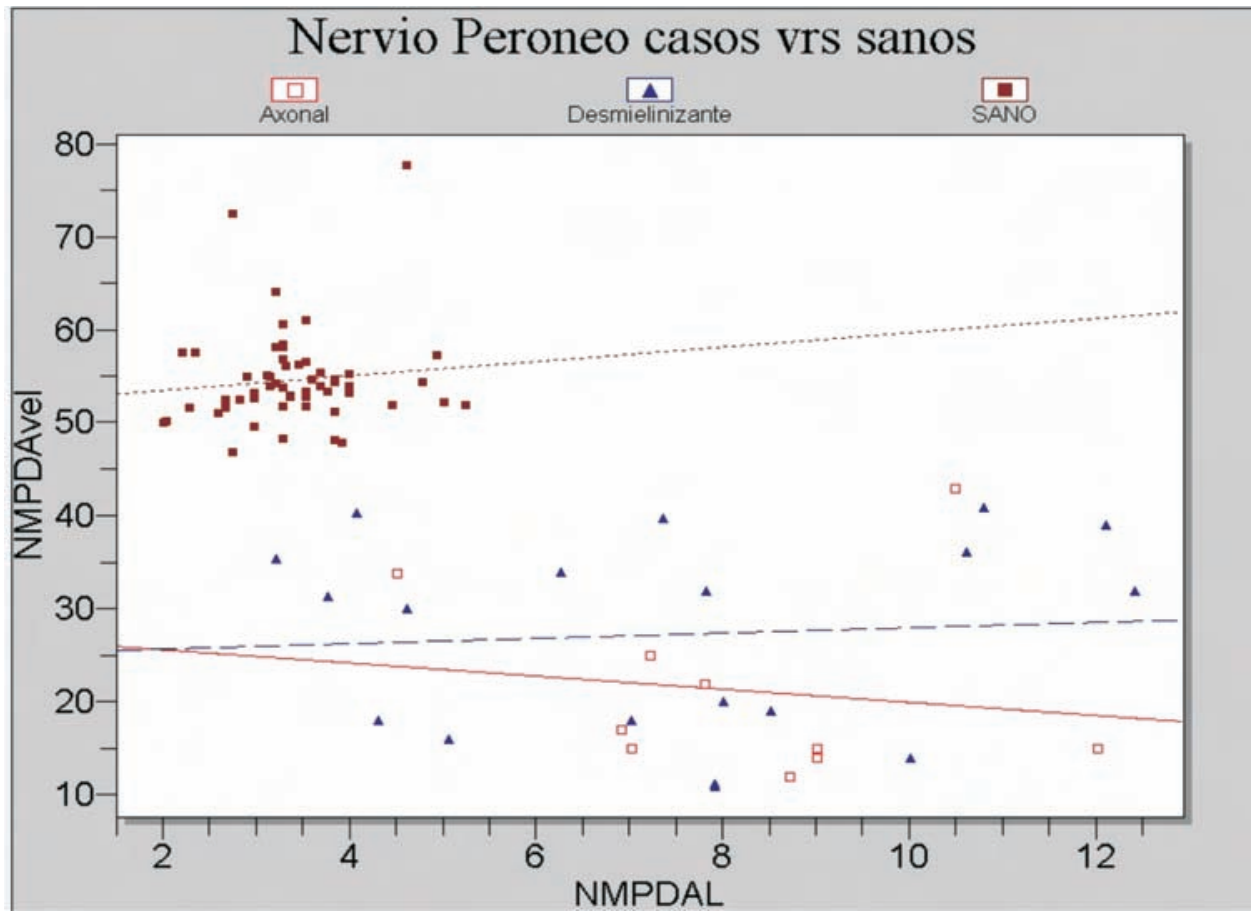

Se observan las latencias y velocidades afectadas en las formas desmielinizantes y axonales presentando mayor afectación de estos parámetros los casos con formas desmielinizantes y predominando amplitudes más bajas en las axonales 
Tabla 4. Conducción de nervios motores: medias y rango mínimo y máximo en pacientes con formas desmielinizantes versus axonales

\begin{tabular}{|c|c|c|c|}
\hline Nervio & Desmielinizante( $n=19)$ & Axonal $(n=10)$ & $P$ \\
\hline \multicolumn{4}{|c|}{ Mediano } \\
\hline Latencia distal & $5.6 \quad(2.66-12)$ & $4.65(3.69-5.6)$ & 0.03866 \\
\hline Amplitud & $1.8 \quad(0-4.89)$ & $0.38(0-3.03)$ & 0.0318 \\
\hline Velocidad & $28.17(32-39)$ & 33 (30-65) & 0.0463 \\
\hline $\begin{array}{l}\text { Latencia Onda } \\
\mathrm{F}\end{array}$ & $33.54(28.27-45.21)$ & NR & 0.003612 \\
\hline \multicolumn{4}{|c|}{ Ulnar } \\
\hline Latencia distal & $4.17(2.03-8.9)$ & $3.61(2.97-4.55)$ & 0.00906 \\
\hline Amplitud & $1.91(0-6.45)$ & $0.9(0-5.45)$ & 0.01730 \\
\hline Velocidad & $42.77(23.0-60.5)$ & $34.18(30-65.3)$ & 0.00460 \\
\hline $\begin{array}{l}\text { Latencia onda } \\
\mathrm{F}\end{array}$ & $36.21(29.2-45.4)$ & NR & 0.00862 \\
\hline \multicolumn{4}{|c|}{ Peroneo } \\
\hline Latencia distal & $6.45(3.2-12.1)$ & $7.26(5.1-10)$ & 0.05415 \\
\hline Amplitud & $1.76(0-7.36)$ & $1.08(0-3.2)$ & 0.045138 \\
\hline $\begin{array}{l}\text { Velocidad de } \\
\text { Cond. }\end{array}$ & $36.76(36.1-42.1)$ & $40.6(32.2-53.1)$ & 0.06186 \\
\hline \multicolumn{4}{|c|}{ Tibial } \\
\hline Latencia distal & $6.1(3.1-10.3)$ & $6.24(5.7-6.7)$ & 0.0733 \\
\hline Amplitud & $3.3(0-12.3)$ & $2.98(0-15)$ & 0.7055 \\
\hline $\begin{array}{l}\text { Velocidad de } \\
\text { Cond. }\end{array}$ & $22.9(17.07-56.24)$ & $13.9(15.1-47.7)$ & 0.2317 \\
\hline $\begin{array}{l}\text { Latencia de } \\
\text { ondaF }\end{array}$ & $39.7(28.3-56.2)$ & NR & 0.0041 \\
\hline
\end{tabular}

Se observan los parámetros distales latencia, velocidad, amplitud de los nervios motores Mediano, Ulnar, Peroneo, Tibial; y la latencia mínima de onda F. NR : Inexcitable. 
Tabla.5 Conducción de nervios sensitivos: medias y rango mínimo y máximo

\begin{tabular}{|l|c|c|c|}
\hline Nervio Sensitivo & AIDP(n=19) & AMAN(n=8) & P \\
\hline \multicolumn{4}{|c|}{ Mediano } \\
\hline Latencia distal & $5.6(2.66-12)$ & $4.65(3.69-5.6)$ & 0.03866 \\
\hline Amplitud & $1.8(0-4.89)$ & $0.38(0-3.03)$ & 0.0318 \\
\hline Velocidad & $28.17(32-39)$ & $33(30-65)$ & 0.0463 \\
\hline \multicolumn{4}{|c|}{ Ulnar } \\
\hline Latencia distal & $4.17(2.03-8.9)$ & $3.61(2.97-4.55)$ & 0.00906 \\
\hline Amplitud & $1.91(0-6.45)$ & $0.9(0-5.45)$ & 0.01730 \\
\hline Velocidad & $42.77(23.0-60.5)$ & $34.18(30-65.3)$ & 0.00460 \\
\hline \multicolumn{4}{|c|}{ Sural } \\
\hline Latencia distal & $6.45(3.2-12.1)$ & $7.26(5.1-10)$ & 0.05415 \\
\hline Amplitud & $1.76(0-7.36)$ & $1.08(0-3.2)$ & 0.045138 \\
\hline Velocidad & $36.76(36.1-42.1)$ & $40.6(32.2-53.1)$ & 0.06186 \\
\hline
\end{tabular}

Valores distales de Latencia, Amplitud, Velocidad para nervios sensitivos mediano, ulnary sural

\section{DISCUSIÓN}

El subtipo de Síndrome de Guillain-Barrè más comúnmente descrito es la polirradiculoneuropatía desmie-linizante inflamatoria aguda (AIDP) $(10,11,12)$. Existen otros subtipos tal como las formas axonales; de estos el subtipo AMAN (Neuropatía axonal motora aguda) presenta déficit motor puro y, cuando también se afectan las fibras sensitivas el subtipo es conocido como neuropatía axonal motora y sensitiva (AMSAN) $(12,14,15)$.

La incidencia de estas categorías varía de región en región, mientras que la incidencia anual promedio de Síndrome de Guillain-Barrè tiene un rango de 0.66 a 4 casos por 100,000 habitantes. Se ha registrado que la frecuencia de AMAN varía entre los países de oeste y Asia de forma considerable; de manera tal que en pacientes estudiados en América y Europa es de menos del 10\%, en países del Norte de China es hasta de 65\% y en Japón de un 40\% $(16,17)$.

Nosotros encontramos los tres subtipos de SGB en sus formas axonales y desmielinizantes; predominando la variante AIDP en un $65 \%(n=19)$ de los casos, la Neuropatía axonal motora aguda representó el 28\% (n=8) de los pacientes 
estudiados y la neuropatía axonal sensitiva motora aguda se obtuvo en 2 (7\%) casos del total. Se presentaron las formas axonales en un total de 10 pacientes y las formas desmielinizantes en 19 casos.

Todos los reportes han coincidido en que se presenta una afectación 1.5 veces mayor en hombres que en mujeres $(18,19)$; coincidiendo con nuestro estudio que de un total de 29 casos se dio una relación masculino: femenino de 17:12; con predominancia en 17 (59\%) de los casos el sexo masculino; 1.4 veces más frecuente en varones

En Europa y Norteamérica la incidencia aumenta constantemente de menos de un caso por 100,000 habitantes menores de 30 años a cuatro en mayores de 75 años. En China se observa la misma incidencia en niños y es mucho menor en adultos (20). En las regiones Caribeñas, por ejemplo en la Isla Curacao la incidencia fue de 1.62 por 100,000 habitantes. En nuestro país se realizó en el período de1989-1999 un estudio que valoró la incidencia de SGB y parálisis flácida en niño, en el Hospital Materno Infantil obteniendo valores de $1.37 / 100,000$ por año para SGB y de 1.89/100,000 habitantes por año de parálisis flácida a nivel nacional y hospitalaria.

La caracterización neurofisiológica de nuestros casos mostró mayor similitud con los datos obtenidos en China, Japón y Sur América que la publicada en Norte América y Europa (21,22); presentándose las formas desmielinizantes en 19 (65\%) y las formas axonales en 10(35\%) caso; ocurriendo en estás ultimas las formas clínicas más severas, mayor compromiso respiratorio, mayor cantidad de días de hospitalización y mayor necesidad de Cuidados Intensivos en relación a los pacientes con formas desmielinizantes(23).

Algunos casos de disautonomía aguda sin compromiso de nervios somáticos pueden ser inflamatorios y posiblemente autoinmunes. Se han relacionado las disautonomías a mayor mortalidad, en nuestros pacientes se presentaron en un $72 \%$ de los casos (24)

Se ha documentado que entre el 50 y el $70 \%$ de los casos de SGB, existe un cuadro infeccioso en las cuatro semanas anteriores, que bien puede ser una infección respiratoria alta o gastroenteritis. En nuestros pacientes se presentó el antecedente de proceso infeccioso en $79 \%$ de los casos, predominando este antecedente entre las formas desmielinizantes. Los procesos respiratorios se presentaron con mayor frecuencia que los gastroentéricos.

Se encontraron asociados otros procesos infecciosos diferentes a los mencionados 
en 2 casos; uno de ellos fue Varicela en un adulto de 43 años quien falleció y cursó con un subtipo axonal; $y$ un segundo caso de 15 años con cuadro de Dengue grupo $B$, que mostró una variante desmielinizante con discapacidad moderada y sin afectación de músculos respiratorios.

La mortalidad de SGB puede ser de un $5 \%$ en centros con cuidados intensivos y de 7 a $15 \%$ en pacientes con daño permanente axonal. En nuestros casos se dio una mortalidad de $7.5 \%$ asociada a la presentación de formas moderadas y severas(24).

\section{CONCLUSIONES}

1. Las formas desmielinizantes de Síndrome de Guillain-Barrè se presentaron en los pacientes estudiados con alta frecuencia. Sin embargo la frecuencia de las formas axonales es superior a lo reportado en América y Europa, y similar a la reportada en Japón y el norte de China

2. Las formas axonales de Síndrome de Guillain-Barrè presentaron las formas clínicas más severas con mayor afectación de músculos respiratorios, asociados a mayor tiempo de hospitalización y mayor discapacidad.

3. Desconocemos las etiologías de las variantes axonales ya que en este estudio no fue posible la realización de pruebas para Campylobacter jejuni que es el agente que más se ha relacionado con formas axonales.

\section{RECOMENDACIONES}

1. Establecer protocolos de seguimiento para todas las formas de Síndrome de Guillain-Barrè sin importar su grado de severidad.

2. Valoración neurofisiológica de todos los pacientes que ingresan con diagnóstico de Síndrome de Guillain-Barrè para determinar si tiene una forma axonal versus desmielinizante y de esta manera mejorar sus probabilidades de recuperación.

3. Continuar estudios Neurofisiológicos para normatizar datos en nuestra población y de esta forma tener datos referenciales de nuestro país.

4. Dada la elevada incidencia del SGB en nuestro país y la incidencia de subtipos axonales se requiere de un incremento de las camas de Cuidados Intensivos en vista de que este tipo de pacientes requirieron del apoyo de estas unidades y por períodos prolongados. 


\section{CONTRIBUCIÓN DE LOSAUTORES}

\section{Dra. Carmen Julissa Aguilar Rosales}

Autor Principal

Participación completa en todos los apartados del presente estudio y responsable por la integridad de los datos, objetividad y certeza de los resultados.

\section{Dr. Marco Tulio Medina Hernández \\ Co-autor. Tutor Principal}

Participación en el diseño del estudio, análisis e interpretación de los datos, revisión de la redacción del manuscrito, supervisión durante todo el proceso de investigación.

\section{Dra. Rebeca Hernández Toranzo:}

Co-autor. Tutora segunda

Participación completa en la evaluación clínica y electrofisiológica, diseño, análisis de datos e interpretación.

\section{Dra. Martha Matamoros:}

Co-autor Asesor y revisora

Participación en análisis e interpretación de datos, corrección de manuscrito

\section{Dra. Claudia Amador.}

Neuróloga participó en la evaluación clínica de pacientes.

\section{Dr. José Angel Lara Pinto:}

MD, MPH, MSc. Co-autor. Asesor enAnálisis Estadístico

\section{AGRADECIMIENTOS}

a) Por el desarrollo del estudio:

A los pacientes y personas sanas que permitieron la realización del estudio neurofisiológico en apoyo a la ciencia, Dr. Theodore Munsat, Dr. Kenton Holden (Revisores Internacionales), Dr.Miguel Solano, Dra. Sofia Dubón, Dr.Arnold Thompson, Dr. José Medardo Lara, Dra. Vanessa Sirias (Médicos Residentes del Postgrado de Neurología 2007-2008), Dr. Sergio Villeda.

b) Personales:

A Dios por permitirnos salud, a mis padres y abuelos, hermanas (Jessica, 
Cinthia, Jenny Aguilar), a mi esposo e hija, a mi casi madre Nidia Girón, personal de enfermería y amigos que colaboraron en la trayectoria de esta etapa de mi vida.

\section{BIBLIOGRAFÍA}

A. Dilley, N.A. Gregson, R.D. M. Hadden, K.J. Smith. Effects on axonal conduccion of anti-ganglioside será and será from patients with Guillain-Barrè syndrome. Journal of neurounmunology. 2003;(139): 133-140.

A. Criado Molina, JL Pérez y MA Frias. Síndrome de Guillain-Barrè prolongado. Anales Pediatría.2003; (58): 74-76.

Alberts Jw, Donofrio, McGonagle TK. Sequential electrodiagnostic abnormalities in acute inflammatory demyelinating polyradiculoneuropathy. Muscle and Nerve. 1985; (8): 528-539.

Andrew C.F. Hui, K.M. Chow, Amy, and S.Y. Tang. Electrophysiological, clinical and epidemiological study of Guillain-Barrè Syndrome in Hong Kong. Journal of Clinical Neuroscience. 2005;(12): 134-136.

Asbury AK and Cornblath DR. Assessment of current diagnostic criteria for Guillain-Barré syndrome. Ann Neurol 1990; 27 (suppl): 21-24

Asbury AK, Arnason BG and Adams RD. The inflammatory lesion in idiopathic polyneuritis. Medicine 1969; (48): 173-215.

C.Y. Gao, T.W. Ho, G.L. Wang,G.H. Zang, J.X. Mao, C.Y.Li, et al. Acute motor axonal neuropathy. In: J.Kimura and R.Kaji, editors. Physiologyof ALS and Related Diseases. Amsterdam: The Netherlands. Elsevier Science.B.V.. 1997; 119-128

Durand, Marie-Christine, Raphaël Porcher, David Orlikowski, Jerome Aboad, Christian Devaux and Bernard Clair. Clinical and electrophysiological predictors of respiratory failure in Guillain-Barré syndrome: a prospective study. Lancet Neurology. 2006; 118-123.

E. Ortiz-Corredor. Factores que influyen en el pronóstico del Síndrome de GuillainBarrè infantil. Rev. Neurol. 2004; 38(6): 518-523.

Gordon Paul H and Wilbourn Asa J. Early electrodiagnostic findings in Guillain-

Barré syndrome. Arch Neurol. 2001; (58): 913-917.

Griffin JW, Li CY and Ho TW. Guillain-Barré Sybdrome in northern China: The spectrum of neuropathologic changes in clinically defined cases. Brain 1995; (118): 577-595.

Guillermo Paradiso, Juan Tripoli and Santiago Galicchio. Epidemiological, clinical and electrodiagnostic findings in childhood Guillain-Barrè Syndrome: a reppraisal. Ann Neurology 1999; (46): 701-707.

Hugh J. Willison and Graham M Ohanlon. The inmunopathogenesis of Miller Fisher syndrome. Journal of neurounmunology. 1999;(100): 3-12 
Hughes RAC and Cornblath DR. Guillain-Barré syndrome. Lancet. 2005; (366): 1653-66.

Hughes Richard A C and Cornblath David R,. Guillain-Barré syndrome. Lancet 2005; (366): 1653-66.

J.P. Monteiro, S. Fonseca, J. Proenca, P.Calhau, M.Braga, y M.J. Fonseca. Síndrome de Guillain-Barré en edad pediátrica. Experiencia de la Unidad de Neuropediatría de un hospital portugués. Rev. Neurología 2006; (42): 144-9.

Kasumi Nagasawa, and Satoshi Kuwabara. Electrophysiological subtypes and prognosis of Childhood Guillain-Barrè Syndrome in Japan. Muscule and Nerve. 2006; (33):766-770.

Kavitha D. Mogal, H. Antony, Monique M. Ryan, et al. The Pharyngeal-CervicalBrachial Form of Guillain-Barré Syndrome in Childhood. Pediatr Neurol. 2005; (33): 285-288.

Kazue Ogawara, Satoshi Kuwabara, Masahiro Mori, and Takamichi Hattori. Axonal Guillain-Barrè Syndrome: Relation to Anti-Ganglioside Antibodies and Campylobacter jejuni Infection in Japan. Ann Neurol 2000; (48): 624-631

Molinero MR, Varon D, and Holden KR. Epidemiology of childhood Guillain-Barrè as a cause of acute flaccid paralysis in Honduras: 1989-1999. J Child Neurology. 2003; (18): 741-747

Nina M. van Sorge, Nobuhiro Yuki C, Michiaki Koga, Keiichiro Susiki, Marc D. Jansen, John H. J. et al. Ganglioside-specific IgG and IgA recruit leukocyte effector functions in Guillain-Barré síndrome. The Netherlands. Journal of Neuroimmunology. 2007; (182):177-184.

Pi-Lien Hung, Wen-Neng Chang and Li-Tung Huan. A Clinical and Electrophysiologic Survey of Childhood Guillain-Barrè Syndrome. Pediatr Neurol. 2004; (30): 86-91.

Preston D.C and Shapiro B.E. Fundamentals of nerve Conduction studies en: Electromyography and Neuromuscular disorders. 2 ed. Phyladelphia:Elsevier; 2005.

Ted M. Burns, M.D. Guillain-Barrè Syndrome. Semin Neurol 2008; (28): 152-167. 